\title{
Are pulsar glitches triggered by a superfluid two-stream instability?
}

\author{
N. Andersson*, G.L. Comer ${ }^{\dagger}$ and R. Prix* \\ ${ }^{*}$ Department of Mathematics, University of Southampton, Southampton SO17 1BJ, UK \\ ${ }^{\dagger}$ Department of Physics, Saint Louis University, St Louis MO63156, USA
}

\begin{abstract}
Mature neutron stars are expected to have several superfluid components. Strong evidence for this is provided by the glitches that have been observed in dozens of pulsars. The underlying idea behind most glitch models is that, as the neutron star crust spins down due to the emission of electromagnetic radiation, the superfluid component lags behind until a critical point is reached and angular momentum is transferred from the superfluid to the crust, leading to the spin-up associated with the glitch. In this Letter we describe a superfluid analogue of the two-stream instability that is well known in plasma physics, and provide evidence that this instability is likely to be relevant for neutron stars. This is a new physical mechanism which may play a key role in explaining the glitch mechanism and which could also prove to be relevant in laboratory experiments on superfluid Helium.
\end{abstract}

Neutron stars are cosmic laboratories of extreme physics [1]. With a mass of roughly one and a half times that of the Sun compressed inside a radius of ten kilometres, they have density several times that of nuclear matter, strong magnetic fields and may spin rapidly. By now nearly 1300 pulsars (rotating neutron stars) have been observed [2]. In fact, estimates based on the local population and the supernova rate suggest that the total number of neutron stars in the galaxy may be as large as $2 \times 10^{8}$. Despite decades of theoretical effort the physics of these compact objects is not well understood. For example, there are open issues concerning the pulsar radiation mechanism and we do not know the detailed supranuclear equation of state. The main reason for the many uncertainties is that the description of these objects requires physics well beyond our laboratory experience.

Certain aspects of neutron star physics are, however, well established. It is clear that the overwhelming majority of these objects must be very cold in the sense that their temperatures are significantly below the Fermi temperatures of neutrons and protons (typically about $10^{12} \mathrm{~K}$ ). Nuclear physics calculations of the transition temperature to superfluidity consistently yield a value of the order of $10^{9} \mathrm{~K}$ for the neutrons and protons. A newly born neutron star is expected to cool below this temperature within a few weeks to months following the supernova explosion. Thus most neutron stars in our galaxy are expected to contain superfluid components [3]. The solid ion crust that forms the outer kilometer or so of the star will be permeated by superfluid neutrons, while the fluid in the core is expected to contain superfluid neutrons and superconducting protons. The deep core may contain exotic phases of matter like hyperons and/or deconfined quarks, which may also become superfluid [4, 何. This means that astrophysical neutron stars provide a manifestation of large scale quantum phenomena, and if we want to understand their dynamics we need to allow for the presence of, potentially weakly coupled, superfluid components.

Even though a superfluid is locally irrotational, it can mimic ordinary fluid rotation by forming an array of vortices (with typical separation smaller than $10^{-11} \mathrm{~m}$ ). In the core of each vortex the superfluidity is destroyed, the particles are in an ordinary fluid state and can thus carry non-zero vorticity. Superfluids have zero viscosity, but dissipative mechanisms can exist when vortices are present. Particularly important is the direct friction between the different fluid components (known as "mutual friction"). Another consequence of the interaction between the fluids is the non-dissipative entrainment effect. It arises because the bare neutrons (or protons) are "dressed" by a polarization cloud of nucleons comprised of both neutrons and protons. Since both types of nucleon contribute to the cloud the momentum of the neutrons, say, is modified so that it is a linear combination of the neutron and proton currents. When one of the nucleon fluids begins to flow it will, through entrainment, induce a momentum in the other. This has the effect that a portion of the protons (and electrons) will be pulled along with the superfluid neutrons that surround each vortex.

Strong observational support for the presence of superfluid components in a neutron star is provided by the so-called glitches [6]: sudden spin-up events where the observed rotation rate (presumably that of the stars crust) may increase by as much as one part in $10^{6}$. The glitches typically have a relaxation time of the order of days to months, which is much longer than can be explained in terms of normal viscosity. Very soon after the first glitch in the Vela pulsar [7], 8] was observed in 1969 it was argued [9] that the long relaxation time indicates the presence of a neutron superfluid. The glitch events, and the associated relaxation, are usually explained in terms of superfluid vortex dynamics. This is natural since the superfluid can only change its rotation rate if the vortices move. The standard model describes a glitch as an event in which a significant number of vortices are suddenly unpinned from the crust nuclei [10], angular momentum is transferred to the crust and the vortices are eventually repinned. In particular, the so-called "vortex creep" model [11] is known to provide an adequate description of the glitch relaxation. The actual mechanism that 
triggers the glitch remains unspecified in virtually all models, however. This may be one of the most important unresolved issues in this area of research.

In this Letter we propose that a superfluid two-stream instability (analogous to that known to be relevant for electrons streaming through ions in plasma physics [12]) plays a key role in triggering pulsar glitches. The key parameter governing the onset of this dynamical instability is the rotational lag between a superfluid component and the rest of the star. The existence of such a lag is generally assumed in most glitch models and there is observational evidence that glitches occur once this lag builds up to a critical level. From a statistical analysis of 48 observed glitches in 18 pulsars, Lyne, Shemar and Graham Smith [6] deduce that the glitch activity depends linearly on the spin-down rate (as one would expect if a glitch is due to the release of a built up rotational difference). They estimate that a typical value for the critical velocity is $\Delta \Omega / \Omega_{\mathrm{p}} \approx 5 \times 10^{-4}$ (where $\Omega_{\mathrm{p}}$ and $\Omega_{\mathrm{n}}$ are the rotation rates of the crust and the superfluid neutrons, respectively, and $\Delta \Omega=\Omega_{\mathrm{n}}-\Omega_{\mathrm{p}}$ represents the extent to which the superfluid lags behind as the crust spins down).

In a recent paper 13] we have studied a superfluid analogue of the two-stream instability. As far as we are aware, this mechanism has not previously been discussed in this context. Yet, it could turn out to be of extreme importance for all superfluid systems that exhibit relative motion. The two-stream instability is analogous to the familiar KelvinHelmholtz instability 14. Its key distinguishing feature is that the two fluids are interpenetrating rather than in contact across an interface. In principle, the instability may operate in any system with two interpenetrating components moving at different rates. Unstable waves are such that they can be associated with a negative energy (in the sense that the energy in the perturbed flow is smaller than the energy of the unperturbed system). For this to be possible the wave must be such that it moves forwards with respect to one of the fluids, but backwards according to an observer riding along with the other fluid. Here we consider a neutron star in which the two fluids rotate at constant rates. A pulsation mode of the star, which is proportional to $e^{i(\omega t+m \varphi)}$, will carry negative energy if the angular velocity of the mode pattern (the "pattern speed") $\sigma_{p}=-\omega / m$ lies in between the two rotation rates, i.e. when $\Omega_{\mathrm{p}}<\sigma_{p}<\Omega_{\mathrm{n}}$, assuming that the superfluid neutrons lag behind as the charged component is being spun down through electromagnetic braking.

The results described in this Letter were borne out of an effort to understand the dynamics of neutron stars with one, or several, superfluid components. The simplest models involve two interpenetrating fluids, eg. the superfluid neutrons in the inner crust and the core and all charged constituents (the ions in the crust, core protons, and all electrons) and the entrainment effect that acts between them [15, 16, 17]. We have recently demonstrated [13 that the two-stream instability may operate in these systems provided that the two fluids are coupled, either "chemically" or via the entrainment. In order to provide support for the idea that the superfluid two-stream instability is relevant for pulsar glitches we consider a model problem of two fluids, allowed to rotate at different rates, inside a thin shell. By assuming that the shell is infinitesimally thin we may ignore radial motion, which means that the system permits only toroidal velocity perturbations. In fact, all oscillation modes of this shell model are closely related to the inertial r-modes of rotating single fluid objects [18. A somewhat laborious calculation [19] leads to the following dispersion relation for oscillation modes of this system

$$
\begin{aligned}
& \left\{l(l+1)\left[1-x_{\mathrm{p}}(1+\varepsilon)\right](\kappa+\Lambda)-2\left(1-x_{\mathrm{p}}\right) \Lambda+x_{\mathrm{p}} \varepsilon(l-1)(l+2)(1-\Lambda)\right\} \\
& \times\{l(l+1)[1-\varepsilon](\kappa+1)-2-\varepsilon(l-1)(l+2)(1-\Lambda)\}-[l(l+1)]^{2} x_{\mathrm{p}} \varepsilon^{2}(\kappa+\Lambda)(\kappa+1)=0 .
\end{aligned}
$$

Here we have assumed that the waves are expanded in standard spherical harmonics $Y_{l}^{m}(\theta, \varphi)$ with integer indices $l$ and $|m| \leq l$. Furthermore, $x_{\mathrm{p}} \leq 0.15$ is the proton fraction, $\kappa=\omega / m \Omega_{\mathrm{p}}$ represents the frequency of oscillation as measured with respect to the rotation rate of the charged component, and we have introduced a dimensionless measure of the relative rotation $\Lambda=\Omega_{\mathrm{n}} / \Omega_{\mathrm{p}}$. The entrainment effect is represented by the coefficient $\varepsilon$, which is expected to lie in the range $0.4 \leq \varepsilon \leq 0.7$ (see Ref. [16] for further discussion). A detailed analysis of the roots of the above dispersion relation shows that complex frequency modes will exist (for any multipole $l$ ) provided that the relative rotation rate $\Lambda$ is sufficiently large.

As a "physically realistic" example, we consider the case when $\Delta \Omega / \Omega_{\mathrm{p}}=5 \times 10^{-4}$ which corresponds to $\Lambda=1.0005$. We then find that we must have $l>65$ in order for the region of instability to overlap with the anticipated range of the proton fraction and the entrainment. This indicates that the instability will operate on length scales shorter than $\pi R / l \approx 500 \mathrm{~m}$ (if we take the shell radius to be $R=10 \mathrm{~km}$, which would be the size of a typical neutron star). The region of instability for this rotational lag and $l=100$ is illustrated in Figure 1. Since our results only depend on the azimuthal index $m$ through the scaling $\operatorname{Im} \omega=m \Omega_{\mathrm{p}} \operatorname{Im} \kappa$, and the dispersion relation does not depend explicitly on $m$, the fastest growth time corresponds to the $m=l$ mode. By analyzing the large $l$ limit of the dispersion relation 


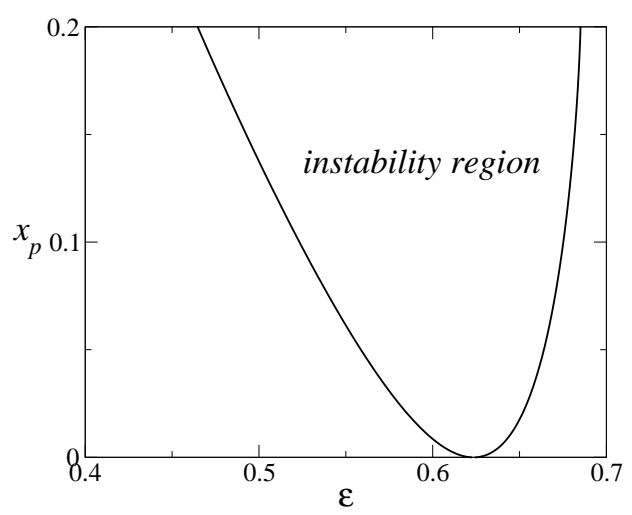

FIG. 1: The two-stream instability region for the case when the superfluid neutrons lag behind the superconducting protons in such a way that $\Delta \Omega / \Omega_{\mathrm{p}}=5 \times 10^{-4}(\Lambda=1.0005)$. The results are for $l=100$, which means that the unstable modes correspond to oscillations on a length scale of a few tens of meters. Similar instability regions exist for all $l>65$. Since typical values for a neutron star core are likely to be such that $x_{\mathrm{p}}<0.15$ and $0.4 \leq \varepsilon \leq 0.7$ one would expect the instability to operate in astrophysical neutron stars.

one finds that the fastest possible growth time for an unstable mode can be approximated by

$$
t \approx \frac{6.7 \times 10^{-2}}{l}\left(\frac{\Delta \Omega}{\Omega_{\mathrm{p}}}\right)^{-1}\left(\frac{P}{0.1 \mathrm{~s}}\right) \mathrm{s}
$$

This simple formula provides a useful estimate of the fastest growth rate of the two-stream instability for different parameter values, and we find that for a star rotating at the rate of the Vela pulsar, $P=89 \mathrm{~ms}$, we would have $t \approx 1.2 \mathrm{~s}$ for $l=m=100$. Modes corresponding to higher multipoles may grow even faster, but as we will discuss later they are also faster damped by viscosity. Interestingly, this predicted growth time is significantly shorter than the resolved rise time of a large Vela glitch [20]: $t<40 \mathrm{~s}$.

Our results suggest that the two-stream instability is generic in dynamical superfluids, and could possibly limit the relative flow in any superfluid system. Furthermore, the results for the shell model problem demonstrate that entrainment provides a sufficiently strong coupling for the instability to operate at astrophysically relevant relative flows. We believe that these results indicate that the two-stream instability is relevant for neutron stars, and that it may be the agent that triggers pulsar glitches. However, much further research is required if we are to understand this new mechanism in detail. For example, it is crucial to establish that the unstable modes grow faster than the relevant dissipation timescales. In the case of a superfluid neutron star core the main dissipation mechanisms are likely to be mutual friction and shear viscosity due to electron-electron scattering. Using estimates due to Mendell (see Eq. 38 in Ref. [21]) one can argue that the shear viscosity will be the dominant dissipation mechanism for all but the lowest values of $l$. To estimate the shear viscosity damping we can use results obtained for the secular r-mode instability [22]. Combining Eq. 43 in Ref. [23] with the viscosity coefficient for electron-electron scattering [22] we estimate the viscous damping time as

$$
t \approx \frac{1.2 \times 10^{4}}{l^{2}}\left(\frac{1.4 M_{\odot}}{M}\right)\left(\frac{R}{10 \mathrm{~km}}\right)^{5}\left(\frac{T}{10^{7} \mathrm{~K}}\right)^{2} \mathrm{~s}
$$

It is interesting to compare this damping timescale to the growth rate of the unstable modes in our shell model. We find that, in order to overcome the viscous damping, an unstable mode must correspond to

$$
l<90\left(\frac{\Delta \Omega / \Omega_{\mathrm{p}}}{5 \times 10^{-4}}\right)\left(\frac{0.1 \mathrm{~s}}{P}\right)\left(\frac{1.4 M_{\odot}}{M}\right)\left(\frac{R}{10 \mathrm{~km}}\right)^{5}\left(\frac{T}{10^{7} \mathrm{~K}}\right)^{2} .
$$

In the case of the Vela pulsar (the archetypal glitching neutron star) the core temperature can be estimated to be $5 \times 10^{7} \mathrm{~K}$ (roughly two orders of magnitude higher than the observed surface temperature 24). At this temperature modes with $l>2500$ or so are likely to be stabilized by shear viscosity. Given that our results indicate that the two-stream instability is active for much smaller values of $l$, see for example Figure 1 , we conclude that dissipation is unlikely to suppress the instability in sufficiently young neutron stars. Naturally, the situation changes as the star cools further. Our estimates show that shear viscosity will suppress all unstable modes if the core temperature is 
below a few times $10^{6} \mathrm{~K}$. Hence, the two-stream instability may not be able to overcome viscosity in a sufficiently cold neutron star. This would be consistent with the absence of glitches in mature pulsars [6].

If the superfluid two-stream instability is, indeed, relevant for pulsar glitches then what is its exact role? Much further work is required to answer this question, but it is interesting to speculate about the possibilities. Most standard models for glitches are based on the idea of catastrophic vortex unpinning in the inner crust [10, 11]. An interesting scenario is the thermally induced glitch model due to Link and Epstein [25]. They show that the deposit of $10^{42} \mathrm{erg}$ of heat in the crust would be sufficient to induce a large Vela glitch. The mechanism that triggers the glitch, eg. by depositing heat in the crust, is typically not identified in these models. We propose that the two-stream instability may fill this gap in the theory. Of course, glitches need not originate in the inner crust. Jones [26] has argued that the vortex pinning is too weak to explain the size and frequency of the Vela glitches. Indeed, the observational evidence for free precession [27] in PSR B1828-11 indicates the absence of significant vortex pinning. If this argument is correct then the large pulsar glitches must be due to some mechanism operating in the core fluid. Since our model problem was based on equations relevant for the conditions that prevail in a neutron star core it is clear that the two-stream instability may equally well serve as a trigger mechanism for glitches originating there. One possibility would be that the unstable mode grows so large that the superfluid degeneracy is broken, which would lead to an immediate coupling to the other normal fluid components. The key requirement for the two-stream instability to operate is the presence of a rotational lag. Such a lag can build up both when there is a strong coupling between the two fluids (i.e. when the vortices are pinned to the crust) and when this coupling is weak [28]. A key issue for future theoretical work on pulsar glitches concerns to what extent a rotational lag can build up in various regions of the star.

Before we conclude this Letter it is worth emphasizing that we expect the two-stream instability to be generic in superfluid systems. The two-fluid equations that we have employed are analogous to the standard Landau model for superfluids, which means that the instability should be relevant also for laboratory systems. It is, in fact, not inconceivable that the notion that this instability triggers pulsar glitches may be tested using experiments on superfluid ${ }^{4} \mathrm{He}$.

NA and RP acknowledge support from the EU Programme 'Improving the Human Research Potential and the Socio-Economic Knowledge Base' (Research Training Network Contract HPRN-CT-2000-00137). NA acknowledges support from the Leverhulme Trust in the form of a prize fellowship. GC acknowledges partial support from NSF grant PHY-0140138.

[1] N.K. Glendenning, Compact stars (Springer, New York 1997)

[2] D.R. Lorimer, Binary and millisecond pulsars at the new Millenium, Living Reviews in Relativity, online article http://www.livingreviews.org/Articles/Volume4/2001-5lorimer

[3] J.A. Sauls, Superfluidity in the interiors of neutron stars, pp. 457-490 in Timing Neutron Stars, Ed: H. Ögelman and E.P.J. van den Heuvel (Kluwer, Dordrecht 1989)

[4] T. Takatsuka and R. Tamagaki, Nucl. Phys. A 670, 222 (2000)

[5] M. Alford, J. Berger and K. Rajagopal, Nucl. Phys. B 571, 269 (2000)

[6] A.G. Lyne, S.L. Shemar and F. Graham Smith, Monthly Notices R Astr. Soc 315534 (2000)

[7] V. Radakrishnan and R.N. Manchester, Nature 222229 (1969)

[8] P.E. Reichley and G.S. Downs, Nature 222229 (1969)

[9] G. Baym, C. Pethick, D. Pines and M. Ruderman, Nature 224872 (1969)

[10] P.W. Anderson and N. Itoh, Nature 25625 (1975)

[11] K.S. Cheng, M.A. Alpar, D. Pines and J. Shaham, Astrop. J. 330835 (1988)

[12] D. Anderson, R. Fedele, and M. Lisak, Am. J. Phys. 691262 (2001)

[13] N. Andersson, G.L. Comer and R. Prix, The superfluid two-stream instability, in preparation (2002)

[14] P.G. Drazin and W.H. Reid, Hydrodynamic stability (Cambridge Univ. Press, New York 1981)

[15] N. Andersson and G.L. Comer, Monthly Notices R Astr. Soc. 328530 (2001)

[16] R. Prix, G.L. Comer, and N. Andersson, Astron. Astrophys. 381178 (2002)

[17] R. Prix and M. Rieutord, Astron. Astrophys. 393, 946 (2002)

[18] J.C.B. Papaloizou and J.E. Pringle, Monthly Notices R Astr. Soc. 182423 (1978)

[19] N. Andersson, G.L. Comer and R. Prix, The inertial modes of rotating superfluid neutron stars, in preparation (2002)

[20] R.G. Dodson, P.M. McCulloch and D.R. Lewis, Astrop. J. Lett. 56485 (2002)

[21] G.L. Mendell, Astrop. J. 380530 (1991)

[22] N. Andersson and K.D. Kokkotas, Int. J. Mod. Phys. D 10, 381 (2001)

[23] K.D. Kokkotas and N. Stergioulas, Astron. Astrophys. 341110 (1999)

[24] D. Page, Thermal evolution of isolated neutron stars, p. 539 in The many faces of neutron stars, Ed: R. Buccheri, J. van Paradijs and M.A. Alpar (Kluwer, Boston 1998) 
[25] B. Link and R.I. Epstein, Astrop. J. 457844 (1996)

[26] P.B. Jones, Monthly Notices R Astr. Soc. 296217 (1998)

[27] I.H. Stairs, A.G. Lyne and S.L. Shemar, Nature 406484 (2000)

[28] D. Langlois, D.M. Sedrakian and B. Carter, Monthly Notices R Astr. Soc 297, 1189 (1998) 